\title{
Intralesional Triamcinolone May Not Be Beneficial for Treating Acute Hidradenitis Suppurativa Lesions: A Double-Blind, Randomized, Placebo-Controlled Trial
}

\author{
Kristen Fajgenbaum, BA, ${ }^{*}$ Lauren Crouse, MD ${ }^{\dagger}$ Li Dong, PhD,${ }^{\ddagger}$ Donglin Zeng, PhD ${ }^{\ddagger}$ and \\ Christopher SAYed, MD $\$$
}

BACKGROUND Hidradenitis suppurativa (HS) is a chronic, inflammatory condition characterized by recurrent nodules, sinus tracts, comedones, and scarring. Hidradenitis suppurativa is often associated with pain and decreased quality of life. Limited clinical trial data exist regarding the management of acute HS lesions, but clinical experience and a prospective case series suggest that intralesional triamcinolone may be useful.

OBJECTIVE To compare the efficacy of intralesional triamcinolone to placebo for the treatment of HS inflammatory lesions.

MATERIALS AND METHODS This is a double-blind, randomized, placebo-controlled trial comparing intralesional triamcinolone $10 \mathrm{mg} / \mathrm{mL}$, triamcinolone $40 \mathrm{mg} / \mathrm{mL}$, and normal saline (NS). Thirty-two subjects at University of North Carolina Dermatology and Skin Cancer Centers were enrolled for a total of 67 lesions. Subjects reported pain scores, days to resolution, and satisfaction on a standardized survey over a 14-day period.

RESULTS When intralesional injections of triamcinolone $10 \mathrm{mg} / \mathrm{mL}$, triamcinolone $40 \mathrm{mg} / \mathrm{mL}$, and NS were compared, no significant difference was found for days to HS inflammatory lesion clearance, pain reduction at Day 5, or patient satisfaction.

CONCLUSION No statistically significant difference was found between varying concentrations of triamcinolone and NS for the treatment of HS lesions. Steroid injections may be less effective for the management of acute HS than typically presumed.

The authors have indicated no significant interest with commercial supporters. Institutional review board approval: reviewed and approved by UNC IRB; approval \#16-0773. Clinicaltrials.gov listing: NCT02781818.

$\mathrm{H}$ idradenitis suppurativa (HS) is a dermatologic condition characterized by painful, recurrent inflammatory nodules and sinus tracts with a prevalence of $0.05 \%$ to $4 \% .^{1,2}$ In severe cases, secondary lesions including rope-like scarring, plaque-like induration, and multihead comedones may be present. ${ }^{3}$ The affected skin is primarily located in the axilla, inframammary and intermammary folds, and the inguinal and anogenital regions. ${ }^{3}$ The average age of onset for HS is in the early twenties with an average delay in diagnosis of 5 to 14 years. ${ }^{3-5}$
In a survey, individuals with HS reported an average of 2 new inflammatory nodules per month, lasting an average of 6.9 days. ${ }^{5}$ In another study, $65 \%$ of patients sought out medical attention for pain control due to HS flares. ${ }^{6}$ When assessing how HS impacts daily life, patients reported that the worst aspects of living with the disease are pain, restriction of daily activities, lack of intimacy due to location of lesions, and lesion malodor. Unfortunately, the hardships associated with HS lead to approximately half of patients experiencing a psychiatric comorbidity

*University of North Carolina at Chapel Hill School of Medicine, Chapel Hill, NC; ${ }^{+}$Department of Internal Medicine, Carolinas Medical Center, Chapel Hill, NC; ${ }^{\ddagger}$ Department of Biostatistics, UNC Gillings School of Global Public Health, Chapel Hill, NC; §Department of Dermatology, University of North Carolina School of Medicine, Chapel Hill, NC; L. Crouse is currently affliated with the Department of Dermatology, University of North Carolina School of Medicine, Chapel Hill, NC 
with an increased risk of depression, anxiety, and suicide. $^{7,8}$

Hidradenitis suppurativa is understood to be an inflammatory disease affecting follicular units, rather than an infectious process. Early lesions characteristically show normal bacterial skin flora, suggesting that bacterial growth in chronically inflamed nodules is a secondary process. ${ }^{9}$ The initial lesions seem to involve perifollicular infiltration of IL-1, IL-10, IL-17, tumor necrosis factor, S100A8, S100A9, and caspase-1. Neutrophils, monocytes, and mast cells are also prevalent. ${ }^{10-15}$ It is proposed that the inflammatory process is perpetuated by the spillage of follicular contents into surrounding tissue.

Tunneling and scar formation occur during the healing process. ${ }^{10}$

Treatment options for HS range from topical antibiotics and monoclonal antibodies to surgical excisions. Data supporting management of HS flares are lacking, but intralesional triamcinolone is commonly used for the treatment of acute lesions. ${ }^{10}$ A national database of patients with HS demonstrated that $6.7 \%$ received triamcinolone for treatment. ${ }^{16}$ Topical steroids were the third most common treatment for HS, behind antibiotics and pain medications. A prospective case series assessed the efficacy of intralesional triamcinolone $10 \mathrm{mg} / \mathrm{mL}$ (TAC10) in the treatment of HS flares. A significant reduction in pain 1 day after injection was found, as well as a decline in erythema, edema, suppuration, and lesion size after 1 week. ${ }^{17}$ The absence of a placebo control was a major limitation of this study. To strengthen the data on intralesional triamcinolone for the treatment of acute HS, a trial was conducted to compare triamcinolone with normal saline (NS) placebo.

\section{Methods}

This is a randomized, double-blind, placebocontrolled trial assessing the efficacy of intralesional triamcinolone at resolving lesions and reducing pain in patients experiencing an acute HS flare. Patients were recruited at HS subspecialty clinics at University of North Carolina at Chapel Hill (UNC) Dermatology and Skin Cancer Centers in Chapel
Hill, NC, and Hillsborough, NC. This study was approved by the UNC Institutional Review Board, and all patients provided written informed consent before enrollment.

\section{Study Population}

Patients at routine office visits were eligible for this study if they were at least 16 years old, experiencing an acutely inflamed nodule, either on no antibiotics or on a stable course for at least 4 weeks before the visit, and had at least a year-long history of HS symptoms. Acceptable antibiotics included topical clindamycin, topical chlorhexidine gluconate, oral doxycycline, oral minocycline, or oral clindamycin with or without rifampin. Patients who were unable to provide informed consent, or informed assent for those younger than 18 years with guardian consent, were excluded from the study. Discrete, painful lesions less than 2 centimeters in size with signs of active inflammation were identified by a dermatologist who specializes in HS (Figure 1). A maximum of 3 nodules per person could be included. At enrollment, information was collected on disease history, and the HS specialist assessed severity of the disease based on Hurley Stage and number of nodules and sinuses in the area to be injected. Hurley 1 disease is limited to inflammatory nodules, Hurley 2 includes disease that has progressed to the formation of sinus tracts, and Hurley 3 involves interconnecting sinus tracts in multiple areas.

\section{Treatment}

The treatment arms were $0.1 \mathrm{~mL}$ of nonbacteriostatic $0.9 \%$ sodium chloride, TAC 10 , and triamcinolone 40 $\mathrm{mg} / \mathrm{mL}$ (TAC40). The volume of $0.1 \mathrm{~mL}$ was chosen because it is consistent with standard practice at UNC

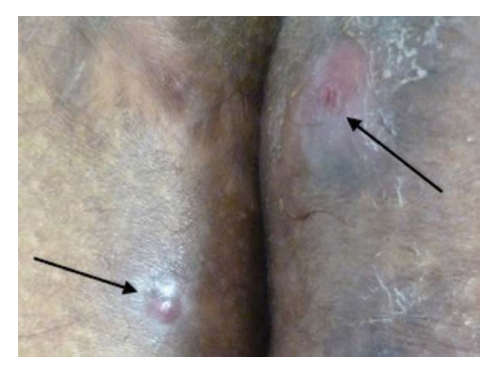

Figure 1. Typical discrete inflammatory nodules included in the study (arrows). 
for treatment of discrete HS lesions and does not create a field effect. Lesions were randomized in a 1:1:1 ratio to one of the treatment arms by the coinvestigator. The coinvestigator prepared syringes and covered them with opaque tape to maintain blinding for subjects and the principal investigator who performed the injections.

Subjects were asked to rate the pain of each randomized lesion on a scale from 1 to 10 before intervention. The subjects received a paper questionnaire to complete for each lesion on posttreatment days 1, 2, 3, 5, 7, 10, and 14. Subjects were instructed to rate the pain associated with each lesion on the specified days and note whether it had resolved. Lesion resolution was defined on the questionnaire as "when the pain is gone and the lesion has returned to how it was before it became inflamed." On Day 14, subjects indicated whether the intervention "made it worse," was "not helpful," "a little bit helpful," "moderately helpful," or "very helpful." Subjects were instructed to mail in their questionnaires after completion.

\section{Sample Size Calculation}

The primary outcomes evaluated were average days to resolution of lesions and reduction of the associated pain 5 days after intervention. Pain reduction was calculated by subtracting the Day 5 pain score from the preintervention pain score. With 60 lesions, the study was powered at $80 \%$ to detect a 3-day difference between NS and the total group of lesions that received triamcinolone (TAC) consisting of TAC10 plus TAC40 and powered at $100 \%$ to detect a 3 -point difference between pain score reduction at Day 5 . With 50 lesions, the power became $64 \%$ and $100 \%$, respectively. In addition, TAC10 versus TAC40 was assessed.

\section{Data Analysis}

SAS 9.4 was used for all analyses. In the descriptive analysis, mean values, $95 \%$ confidence intervals (CI), and $p$-values were calculated for NS, TAC10, TAC40, and TAC for each outcome. Survival analysis based on the gamma frailty model was used to compare days to resolution. In the multivariate analysis, linear mixedeffect models were used for the outcomes of pain reduction and satisfaction to assess and account for potential correlation with demographics, lesions treated in the same patient, location of nodules, number of sinuses and nodules in the injected region, and Hurley stage.

\section{Results}

\section{Subjects}

Between June 2016 and May 2017, 32 patients were enrolled for a total of 67 lesions. In all, 21 lesions were enrolled in the NS arm, 24 lesions were enrolled in the TAC10 arm, and 22 lesions were enrolled in the TAC40 arm (Figure 2). Five subjects were lost to follow-up, which resulted in loss of data for 9 lesions. Fifty-eight lesions had at least partial completion of the survey and were ultimately included in the analysis.

The participants were 36 years old on average and 95\% female, 50\% African American, 45\% Caucasian, and $5 \%$ Hispanic. Literature suggests that the highest disease prevalence is among women and African Americans. ${ }^{1}$ Subjects had an average disease duration of 13.5 years and Hurley Stage of 2.25. Baseline characteristics were similar across interventions and had no significant effect on the outcomes. Moderate correlations were found among lesions on the same subject, so this was accounted for in the analysis.

\section{Lesion Resolution}

Lesions injected with NS had an average resolution of 9.35 days (95\% CI: 6.87-11.83), TAC10 averaged 10.78 days (95\% CI: 8.16-13.4), TAC40 averaged 10.85 days (95\% CI: 8.32-12.38), and TAC averaged

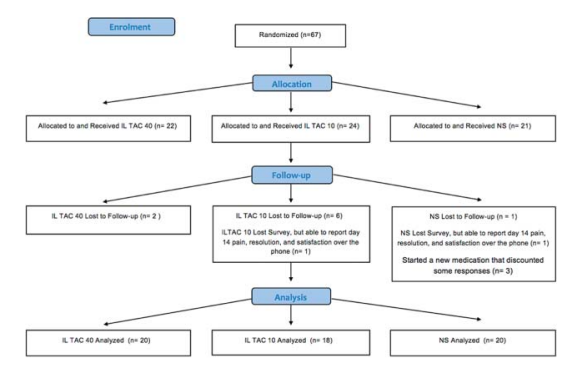

Figure 2. Enrollment and analysis flow diagram. 
10.82 days (95\% CI: 9.26-12.38). When comparing TAC with NS, there was no statistically significant difference between the interventions, $p=.44$

(Figure 3).

\section{Pain Reduction}

Five days after intervention, lesions injected with NS had an average pain score reduction of 2.63 (95\% CI: $1.40-3.86)$, IL TAC 10 had a 2.00 reduction (95\% CI: 0.65-3.35), IL TAC 40 had a 2.30 reduction (95\% CI: 1.14-3.46), and TAC arm had a reduction of 2.16 (95\% CI: 1.32-3.00). When comparing TAC with NS, there was no statistically significant difference, $p=.77$ (Figure 4). All interventions followed a very similar downward trend in pain over the 14 days (Figure 5).

\section{Patient Satisfaction}

All interventions were typically found to be "a little bit helpful" to "moderately helpful" (Figure 6). When comparing TAC with NS, there was no statistically significant difference, $p=.54$.

\section{Adverse Events}

No subjects reported adverse events.

\section{Discussion}

Contrary to the authors' expectations, this study was unable to find a statistically significant difference between $0.1 \mathrm{~mL}$ of TAC and NS for the

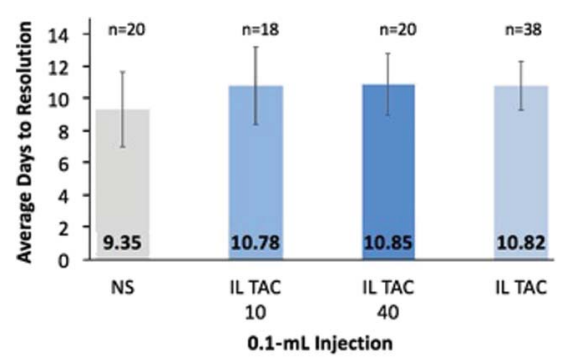

Figure 3. Average days to resolution after injection for normal saline, intralesional triamcinolone $10 \mathrm{mg} / \mathrm{mL}$, intralesional triamcinolone $40 \mathrm{mg} / \mathrm{mL}$, and the average of intralesional triamcinolone $10 \mathrm{mg} / \mathrm{mL}$ and intralesional triamcinolone $40 \mathrm{mg} / \mathrm{mL}$. IL TAC 10, intralesional triamcinolone $10 \mathrm{mg} / \mathrm{mL}$; IL TAC 40, intralesional triamcinolone $40 \mathrm{mg} / \mathrm{mL}$; IL TAC, intralesional triamcinolone; NS, normal saline.

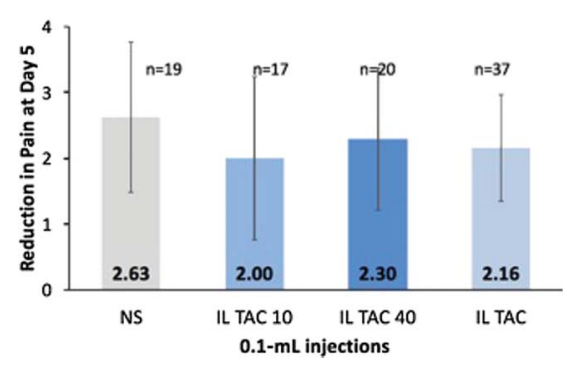

Figure 4. Mean pain reduction 5 days after injection for normal saline, intralesional triamcinolone $10 \mathrm{mg} / \mathrm{mL}$, intralesional triamcinolone $40 \mathrm{mg} / \mathrm{mL}$, and the average of intralesional triamcinolone $10 \mathrm{mg} / \mathrm{mL}$ and intralesional triamcinolone $40 \mathrm{mg} / \mathrm{mL}$. IL TAC 10, intralesional triamcinolone $10 \mathrm{mg} / \mathrm{mL}$; IL TAC 40, intralesional triamcinolone $40 \mathrm{mg} / \mathrm{mL}$; IL TAC, intralesional triamcinolone; NS, normal saline.

treatment of acute HS lesions over a 14-day period. There was also no difference found between TAC10 and TAC40. Since subjects on average found injections to be at least "a little bit helpful," it is possible that the act of puncturing a lesion or instilling external solution provides some relief. Average resolution of lesions and the pain score trends were very similar across interventions, which suggests that lesions have a natural course of resolution. This is consistent with findings in a previous prospective study that demonstrated improvement of pain in the days following treatment with TAC10. ${ }^{7}$ Moving forward, it would be helpful to note the number of days that a lesion has been inflamed before intervention to see if that influences the degree of benefit patients receive from injections.

A limitation of the study is that the number of injections and volume used may not reflect other clinicians' typical use. Benefits seen anecdotally may be due to an injection of higher overall volumes. In addition, data

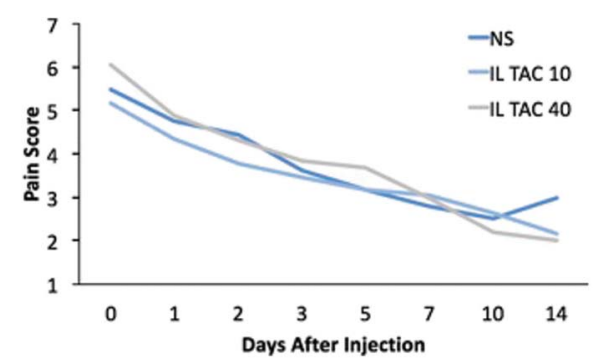

Figure 5. Pain trend using average pain scores on $0,1,2,3$, $5,7,10$, and 14 days after intervention. IL TAC 10, intralesional triamcinolone $10 \mathrm{mg} / \mathrm{mL}$; IL TAC 40, intralesional triamcinolone $40 \mathrm{mg} / \mathrm{mL}$; NS, normal saline. 


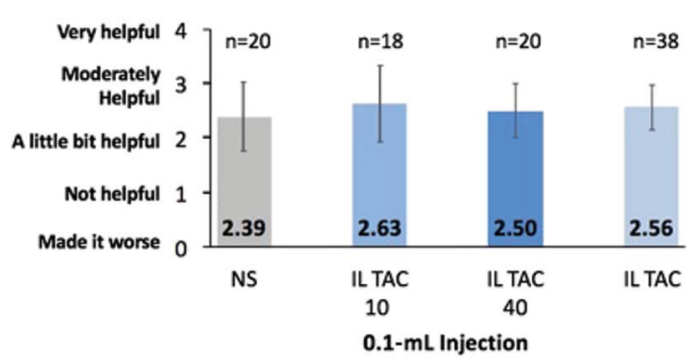

Figure 6. Patient satisfaction 14 days after injection for normal saline, intralesional triamcinolone $10 \mathrm{mg} / \mathrm{mL}$, intralesional triamcinolone $40 \mathrm{mg} / \mathrm{mL}$, and the average of intralesional triamcinolone $10 \mathrm{mg} / \mathrm{mL}$ and intralesional triamcinolone $40 \mathrm{mg} / \mathrm{mL}$. IL TAC 10 , intralesional triamcinolone $10 \mathrm{mg} / \mathrm{mL}$; IL TAC 40, intralesional triamcinolone $40 \mathrm{mg} / \mathrm{mL}$; IL TAC, intralesional triamcinolone; NS, normal saline.

were collected on 58 lesions rather than 60 due to time constraints and loss to follow-up. Although this was slightly below the goal, the study remained wellpowered to detect improvement in pain scores and moderately powered to identify improvement in days to lesion resolution.

Considering the results and potential discomfort, side effects, time, and monetary cost, TAC may not be justified for all patients with HS. Physicians should be guided by their clinical judgment and individual patient's preferences. Larger studies with varying volumes of TAC should be considered in the future.

\section{References}

1. Garg A, Kirby JS, Lavian J, Lin G, et al. Sex- and age-adjusted population analysis of prevalence estimates for hidradenitis suppurativa in the United States. JAMA Dermatol 2017;153: $760-4$.

2. Jemec GB, Heidenheim M, Nielsen NH. The prevalence of hidradenitis suppurativa and its potential precursor lesions. J Am Acad Dermatol 1996;35:191-4.

3. Jemec GB. Clinical practice. Hidradenitis suppurativa. N Engl J Med 2012;366:158-64.
4. Jemec GB, Kimball AB. Hidradenitis suppurativa: epidemiology and scope of the problem. J Am Acad Dermatol 2015;73:S4-7.

5. Von der Werth JM, Williams HC. The natural history of hidradenitis suppurativa. J Eur Acad Dermatol Venereol 2000;14:389-92.

6. Ring HC, Theut Riis P, Miller IM, Saunte DM, et al. Self-reported pain management in hidradenitis suppurativa. Br J Dermatol 2016; 174:909-11

7. Kirby JS, Butt M, Esmann S, Jemec GBE. Association of resilience with depression and health-related quality of life for patients with hidradenitis suppurativa. JAMA Dermatol 2017;153: 1263-9.

8. Thorlacius L, Cohen AD, Gislason GH, Jemec GBE, et al. Increased suicide risk in patients with hidradenitis suppurativa. J Invest Dermatol 2018;138:52-7.

9. Ring HC, Bay L, Kallenbach K, Miller IM, et al. Normal skin microbiota is altered in pre-clinical hidradenitis suppurativa. Acta Derm Venereol 2017;97:208-13.

10. Matusiak L, Bieniek A, Szepietowski JC. Increased serum tumor necrosis factor- $\alpha$ in hidradenitis suppurativa patients: is there a basis for treatment with anti-tumor necrosis factor- $\alpha$ agents? Acta Derm Venereol 2009;89:601-3.

11. Saunte DML, Jemec GBE. Hidradenitis suppurativa: advances in diagnosis and treatment. JAMA 2017;318:2019-32.

12. Prens E, Deckers I. Pathophysiology of hidradenitis suppurativa: an update. J Am Acad Dermatol 2015;73:S8-11.

13. van der Zee HH, de Ruiter L, van den Broecke DG, Dik WA, et al. Elevated levels of tumor necrosis factor (TNF)- $\alpha$, interleukin (IL)- $1 \beta$ and IL-10 in hidradenitis suppurativa skin: a rationale for targeting TNF- $\alpha$ and IL-1 $\beta$. Br J Dermatol 2011;164:1292-8.

14. Hotz C, Bonioto M, Guguin A, Surenaud M, et al. Intrinsic defect in keratinocyte function leads to inflammation in hidradenitis suppurativa. J Invest Dermatol 2016;136:1768-80.

15. Kelly G, Hughes R, McGarry T, van den Born M, et al. Dysregulated cytokine expression in lesional and nonlesional skin in hidradenitis suppurativa. Br J Dermatol 2015;173:1431-9.

16. Davis SA, Lin HC, Balkrishnan R, Feldman SR. Hidradenitis suppurativa management in the United States: an analysis of the national ambulatory medical care survey and MarketScan medicaid databases. Skin Appendage Disord 2015;1:65-73.

17. Riis PT, Boer J, Prens EP, Saunte DM, et al. Intralesional triamcinolone for flares of hidradenitis suppurativa: a case series. J Am Acad Dermatol 2016;75:1151-5.

Address correspondence and reprint requests to: Christopher Sayed, MD, Department of Dermatology, University of North Carolina School of Medicine, 410 Market Street, \#400, Chapel Hill, NC 27516, or e-mail: Christopher_sayed@med.unc.edu 